\title{
Evaluation of the Possibility of Performing the Complex of Works Based on the Models of Their Interaction
}

\author{
Oleg B. Anikin ${ }^{1, *}$, VeraV. Vodyanova ${ }^{2}$, Boris A. Anikin ${ }^{1}$, Nikolay I. Zaichkin ${ }^{3}$ \\ ${ }^{1}$ State University of Management, Moscow, Russia \\ ${ }^{2}$ Economic and Mathematical Methodology Centre (National Institute for Development (Department of Social Sciences, \\ Russian Academy of Sciences)), Moscow, Russia \\ ${ }^{3}$ The Russian Presidential Academy of National Economy and Public Administration (The Presidential Academy, \\ RANEPA), Moscow, Russia \\ *Corresponding author. Email: anikin-oleg-b@mail.ru.
}

\begin{abstract}
The article proposes a mathematical model of the interaction of work performed in an organization at the same time and using common resources. The purpose of the study is the creation of tools with which the decision maker can qualitatively assess the physical ability to execute his orders. As a working hypothesis, it is assumed that the process of performing work is a saturation process, therefore its basic description can be given in the form of a logistic function. Simultaneous performance of work implies their possible influence on each other, manifested in the form of a struggle for common resources. The construction of a mathematical model was associated with the solution of the following problems: selection of a mathematical apparatus that would allow us to evaluate the very possibility of convergence of the described process and present the results at the level of not quantitative but qualitative assessments; identification of the main significant characteristics of the process; with the semantic content of the parameters of the dimensionless model; with an adequate interpretation of the results obtained during the simulation. The empirical material was twenty-year observations of the organization of the managerial process in a higher educational institution, which made it possible to identify a number of factors affecting the quality of management. The model is a system of differential equations, the analysis of which is carried out on the basis of the qualitative theory of differential equations, and allows you to evaluate the qualitative effects that arise when performing a set of works taking into account the level of control available in the organization. On the basis of the basic model, all possible combinations of the agreed limitations in the interaction of the work were considered, as a result of which twelve modifications were constructed. For each model, an analysis of singular points was carried out, and the conditions for the appearance of attractors were revealed. Based on the results of the analysis, recommendations are formulated to help the leader in developing management in solving simultaneous multi-process tasks. The field of research was the sphere of development, managerial decision making and their implementation in a hierarchical organization. Further studies are related to studying the possibilities of increasing the dimensionality of the model and taking into account the factor of employee interest in it, which in the future will lead to the formation of motivation, modeling the enthusiasm of executives and managers, as well as growth points in the organization.
\end{abstract}

Keywords: managerial decision, qualitative theory of differential equations, attractor, phase portrait, dynamical system, singular point, logistic equation, soft modeling

\section{INTRODUCTION}

It is known that the more complex the socio-economic system (not the army), the more connections it has, objective and subjective, the more difficult it is to manage. There is a threshold for effective management of such a system, the definition of which is a non-trivial task. This problem can be partially solved thanks to the tools offered by modern interdisciplinary approaches in General and mathematical modeling. Analysis of the behavior of socio-economic systems based on modeling allows us to avoid possible destructive consequences of a full-scale experiment associated with the irreversibility of processes occurring in such systems; to justify the feasibility of 
reorganization and restructuring of management objects and minimize the associated costs; to identify bottlenecks in the mechanisms of formation of socio-economic systems in the selected areas of economic activity and management, to build the best management technologies in the sense of the set criteria, as well as to solve an impressive number of other equally important tasks.

Management science has always attracted the attention of scientists, researchers, and practitioners $[1,2]$. Attempts to fully share human experience in the management of socioeconomic systems continue to this day. The need for an adequate description and analysis of ongoing processes gave rise to the concept of a system approach, which formed a request for appropriate tools [3, 4]. At the present stage of society's development, only mathematics can provide such tools. The well-known saying of Karl Marx that science only reaches perfection when it begins to use mathematics is widely confirmed in practice in relation to management $[5,6,7]$.

Since quantitative analysis among management representatives often makes it difficult to get a correct idea of the state of the system, it is useful to use qualitative assessments for development and decisionmaking. Qualitative assessments are clear, and they are also good in that they allow you to build strategic trajectories of the organization's development.

The most important feature of a dynamic system is the variability of its state over time [7,8]. The decision-maker must understand how the decision made today will affect the development of the organization in the future and over a certain period of time. Very useful approaches to these issues have been proposed by well-known scientists in the field of management [4, 9, 10]. However, General recommendations always require careful operational elaboration, since a specific management decision is developed and made in a specific organization, located in a specific environment. Therefore, it is very important to give management a tool that provides a qualitative level of rational justification for the development and decisionmaking. This goal is best served by a research area known as soft modeling. In the Humanities, in particular, in the socio-economic sphere, in contrast to exact disciplines, where the laws that determine the course of the studied phenomena are known, which allows them to be described fairly accurately in formal language, it is often important to test certain hypotheses. This usually focuses on qualitative effects. Such models can play the role of simple objects that demonstrate the desired quality behavior. Therefore, when soft modeling of various phenomena in Economics, medicine, and the analysis of natural and man-made disasters, the tools of disaster theory, the theory of dynamical systems on the plane, and one-dimensional maps are widely used. In addition, wellknown nonlinear models that have appeared in one area can sometimes be used as a kind of blocks in other disciplines [5].

The authors propose a model of interaction of work performed in the organization simultaneously and using shared resources. The model is based on the principles of "soft modeling", it is a system of differential equations, the behavior of which is analyzed based on the qualitative theory of differential equations. The scientific novelty consists in the application of nonlinear dynamics methods to the description of the work performance process in the organization. The theoretical significance of the model is that it makes it possible to consider the role of the performer in the implementation of the decision.

\section{MATERIALS AND METHODS}

The basis for qualitative analysis of the behavior of a dynamical system is the qualitative theory of differential equations, which allows us to evaluate the solutions of the system model without finding them explicitly. The main ideas of this theory were laid down by A. Poincare and A. A. Andronov at the turn of the XIX-XX centuries and subsequently developed by their followers in the field of differential equations and system analysis. The basic concepts of the theory are related to special points (rest points, stationary points) of the system, phase portraits of the solution of the system, with the qualities that they may possess, and with those judgments about the behavior and state of the system that can be made on the basis of the detection of certain qualities. Let us have an arbitrary linear system on the plane:

$\dot{x}=A x, \quad x \in R^{2}, \quad A: R^{2} \rightarrow R^{2},(1)$

for which $\lambda_{1}, \lambda_{2}$ - the roots of the characteristic equation. The classification of the types of singular points of such a system is known $[5,8]$.

The concept of equilibrium positions, or stationarity, is extremely important for getting an idea of the behavior of the system, because their presence distinguishes special zones in the field of phase velocity, which is "a local law of evolution of the process, and the theory of ordinary differential equations should, knowing this local law of evolution, recall the past and predict the future" [8]. These special zones are closely related to the concept of process stability, and the definition of their quality in the sense of stability/instability allows us to talk about the possibility of predicting and building a forecast of the system behavior. For a system of two linear differential equations, it is easy to see that the coefficients of the characteristic polynomial of the system are completely determined by the matrix of its operator. The classification of the roots of the characteristic equation constructed in this way is schematically presented in the figure. Based on the above diagram, you can determine the type of special point of the system and relate it to the purpose of the study by obtaining formal dependencies of parameters in the model.

In the General case of describing a system, nonlinear differential equations arise. To study them, a linearization process is performed in the vicinity of special points. Linearization is the rejection of nonlinear terms in the 
Taylor decomposition obtained in the vicinity of a singular point. Conclusions about the behavior of the system in the vicinity of a particular point are made on the basis of the stability principle of the linearized system. The study was conducted for more than 20 years at a higher educational institution - the State University of management. The main interest was in ways to organize the management process in combination with the management style chosen by each new management team. There were 6 such teams, and 5 of them changed over the last 10 years.

\section{DISCUSSION}

Mathematical modeling is increasingly penetrating the Humanities and forces researchers to create synthetic approaches to solving problems in systems of great complexity, where people are important elements and sources of uncertainty. A review of the application of mathematical models has shown their growing relevance in describing nonlinear processes in socio-economic and biological systems [5, 11-14]. The ideology of soft modeling is actively used in describing economic processes $[15,16]$, in describing decision-making processes [17-20], in describing the evolution of systems $[21,22]$, in describing the behavior of groups of people $[23,24]$, in describing information transfer processes [25]. Today is the time of interdisciplinary, so each model built on the fundamental tools of modern mathematics and describing the behavior of a complex system, as a rule, has no analogues and can be considered as a pioneer. For example, in [15], the classical ecological model was transferred to the "consumer-resource" space, and in [18], multi-agent modeling is used for decision-making. The variety of combinations of methods and approaches makes each model unique, so questions of comparing the quality and adequacy of models make sense only in the context of simulated situations.

\section{RESULTS}

Increasing information volumes and accelerating the pace of life creates the need for rapid response to changes in the activities of any organization. The effectiveness of such a response depends directly on the quality of decisions made in the organization. It is very often necessary to perform several tasks simultaneously.

Then the work can interfere with each other, which leads to external interference. This is usually due to the struggle for a resource. But interference can also be internal. The nature of these hindrances lies, on the one hand, in the organization of the management process in the system, on the other hand-in the "quality" of the performers.

Let the system generate an order that must be completed by a certain deadline. We will consider the order execution process as a transition process between two system modes: the initial mode - the order is received, but it has not yet been executed, and the final mode - the order is executed.

The dynamics of this process are well described by the logistics function

$\dot{x}=\alpha x-\beta x^{2}(2)$

where $x$ - the amount of work that needs to be done within the framework of the task presented, $\alpha$ - the productivity with which the work is performed, $\alpha>0$, $\beta$ - the coefficient of "internal interference", reflecting the impact of the need to make clarifications/clarification/approval for this work, $\beta>0$. The Logistics function has two special points: $x=0$ and $x=\frac{\alpha}{\beta}$. The presence of these points and the appearance of the logistics curve allow us to build an analogy with the described process.

In the case when everything is clear and no agreement is needed $(\beta=0)$, the logistic equation turns into the Malthus equation

$\dot{x}=\alpha x(3)$

The solution of which is an exponential function. In this case, the solution is executed on time and at the appropriate level only by selecting the performance indicator $\alpha>0$. Based on this model, with a known amount of work and known deadlines, you can estimate the first approximation of the productivity that the performer should work with. But, as noted above, there may be interference of various nature when performing work. The models presented below assume that such interference exists.

Consider internal interference. A reasonable Manager understands that in order to ensure high-quality execution of the order, the performer must have sufficient time and sufficient forces/capabilities/abilities [10]. Therefore, a reasonable Manager has an ideal model for executing his order:

$$
\dot{x}=\alpha^{\text {ideal }} x-\beta^{\text {ideal }} x^{2} \text { (4) }
$$

where $\alpha^{\text {ideal }}$ is the ideal performance with which the work is performed, $\beta^{\text {ideal }}$ is the ideal coefficient that reflects the acceptable level of deviations from the performance of work for clarification/clarification/approval. At the same time $\alpha^{\text {ideal }} \sim \frac{1}{T^{\text {ideal }}}$

The higher the performance indicator of the $\alpha$ for a fixed $\beta$, the steeper the logistics curve, and the faster the system will move from the initial state to the desired one (in the context of executing an order). 
The higher the $\beta$ deviation rate for a fixed $\alpha$, the lower the horizontal asymptote of the logistics curve is. This means that the system will not reach the specified level of order execution, especially on time.

As a result, in the logistic description of the solution implementation process, we have the following: at $\frac{\alpha}{\beta} \geq \frac{\alpha^{\text {ideal }}}{\beta^{\text {ideal }}}$, the solution will be executed at the required level, but depending on the relationship of the model parameters to the ideal parameters, either exactly on time $T^{\text {ideal }}$, or before the deadline, or later; at $\frac{\alpha}{\beta}<\frac{\alpha^{\text {ideal }}}{\beta^{\text {ideal }}}$, the desired level of execution is not achievable.

Now consider the external interference. As noted above, resource constraints within the system can lead to competition for resources. To describe such "competition" in soft models, a summand of the form is usually used $2 x y$, where $x, y$ - the amount of work to be done in the framework of the task presented, $\gamma$ - the coefficient of interaction of these works [5].

Our task is to make recommendations to managers on reasonable approaches to developing management decisions in terms of implementing a set of works/projects that require simultaneous completion of two works based on the results of considering all possible combinations of external and internal obstacles that affect the intensity of the task. It is obvious that the concept of "two jobs" can be interpreted very broadly and is determined by the degree of aggregation of tasks. All models are described further in the terms entered above.

The interaction model is a global view of the behavior of a set of objects, whereas the state model is a reduced representation of the individual behavior of objects. To fully describe the behavior, you need both models. They complement each other, allowing you to view behavior from two different perspectives. Interactions can be modeled at different levels of abstraction. At the highest level, the system's interaction with external actors is described by use cases. Each use case describes an element of functionality provided by the system to its users. Use cases are useful for representing informal requirements in the model.

The practice has developed a number of approaches to conducting organizational analysis, but the most widespread is the engineering approach. Organizational analysis of the company with this approach is carried out according to a certain scheme using the full business model of the company. The company is considered as a target, open, socio-economic system that belongs to a hierarchical set of open external subsystems (market, government agencies, etc.) and internal subsystems (departments, workshops, teams, etc.). The company's capabilities are determined by the characteristics of its structural divisions and the organization of their interaction.

Obviously, there must be at least two participants in the process to fight for the resource. In General, the model of interaction between the two works looks like this:

$$
\left\{\begin{array}{l}
\dot{x}=\alpha x-\beta x^{2}+\gamma x y, \\
\dot{y}=\delta y-\varepsilon y^{2}+\varphi x y,
\end{array}\right.
$$

where $x, y$ - the amount of work to be done in the framework of the task presented; $\alpha, \delta$ - the performance with which the work is performed with the volumes $x, y$, respectively, $\alpha>0, \delta>0 ; \beta, \varepsilon$ the coefficients of "internal interference", $\beta>0$, $\varepsilon>0, \gamma, \varphi$ - the coefficients of "external interference" (the sign of the coefficients can be any).

Our task is to make recommendations to managers on reasonable approaches to developing management decisions in terms of implementing a set of works/projects that require simultaneous completion of two works based on the results of considering all possible combinations of external and internal obstacles that affect the intensity of the task. It is obvious that the concept of "two jobs" can be interpreted very broadly and is determined by the degree of aggregation of tasks. All models are described further in the terms entered above.

\section{Model 1}

Let there be simultaneous separate performance of two works that do not interfere with each other. For example, these are different jobs and they are performed by different people. Execution processes do not overlap. The performance of the work is determined only by performance and "internal interference" caused by the need to clarify/clarify/agree on this work.

It is assumed that time and resources are sufficient, so the work is equally high-quality, but can be performed both in parallel and sequentially:

$$
\left\{\begin{array}{l}
\dot{x}=\alpha x-\beta x^{2} \\
\dot{y}=\gamma y-\delta y^{2}
\end{array}\right.
$$

The system has a single attractor-a point $\left(\frac{\alpha}{\beta} ; \frac{\gamma}{\delta}\right)$. The coordinates of this point consist of stable values of each of the logistic equations included in the system. Since both equations tend to their stable state, it can be argued that in the organization of work described in the model, the work will be completed on time and at the required level, which means timely and high-quality performance of the overall task.

Model 2

Now let the work interfere with each other, because they require only one thing-the temporary use of a resource. At the same time, none of the work performed receives a 
prize in the resource at the expense of the other work. For example, a single performer performs several types of work that must be done in a time interval that is not sufficient for their sequential execution:

$\left\{\begin{array}{l}\dot{x}=\alpha x-\gamma x y \\ \dot{y}=\delta y-\phi x y\end{array}\right.$

There are only two special points in the model: the trivial $(0 ; 0)$ and $\left(\frac{\delta}{\varphi} ; \frac{\alpha}{\gamma}\right)$. None of these points is an attractor. A special point $\left(\frac{\delta}{\varphi} ; \frac{\alpha}{\gamma}\right)$ that corresponds to the performance of a common task that includes both tasks is of the "saddle"type. This means that the requirements for the overall task will not be fulfilled: both the deadlines and the quality of execution may be violated.

\section{Model 2A}

The work interferes with each other because it requires simultaneous use of a resource. In this case, one of the performed works gets a prize in the resource at the expense of the other work. For example, some performers are distracted from doing the work $x$ in favor of the execution of the work $y$ :

$\left\{\begin{array}{l}\dot{x}=\alpha x-\gamma x y, \\ \dot{y}=\delta y+\phi x y .\end{array}\right.$

There are two special points in the model: a trivial $(0 ; 0)$ and a non-trivial $\left(-\frac{\delta}{\varphi} ; \frac{\alpha}{\gamma}\right)$. None of these points is an attractor. A non-trivial special point of the $\left(-\frac{\delta}{\varphi} ; \frac{\alpha}{\gamma}\right)$ is of the "saddle" type.

\section{Model 3}

Let the work interfere with each other, because they require simultaneous use of a resource. At the same time, none of the work performed receives a prize in the resource at the expense of the other work. In addition, when performing work, there are "internal hindrances" due to the need to make clarifications/clarification/approval for this work.

Since we are interested in performing a set of works, the special points $(0 ; 0),\left(0 ; \frac{\delta}{\varepsilon}\right),\left(\frac{\alpha}{\beta} ; 0\right)$, can be considered trivial. We should be interested in points where both coordinates are positive.

This means that both jobs can be performed simultaneously. As the desired result, we are satisfied with a point $\left(\frac{\alpha \varepsilon-\gamma \delta}{\beta \varepsilon-\gamma \varphi} ; \frac{\beta \delta-\alpha \varphi}{\beta \varepsilon-\gamma \varphi}\right)$ provided that the numerators and denominator of the coordinates are positive. In this case, the solution of the system is stable and makes physical sense. The system has an attractor. This attractor is conditional because

$$
\left\{\begin{array} { l } 
{ \alpha \varepsilon - \gamma \delta > 0 } \\
{ \beta \delta - \alpha \varphi > 0 } \\
{ \beta \varepsilon - \gamma \varphi > 0 }
\end{array} \Leftrightarrow \left\{\begin{array} { l } 
{ \alpha \varepsilon > \gamma \delta } \\
{ \beta \delta > \alpha \varphi } \\
{ \beta \varepsilon > \gamma \varphi }
\end{array} \Leftrightarrow \left\{\begin{array}{l}
\frac{\alpha}{\delta}>\frac{\gamma}{\varepsilon} \\
\frac{\beta}{\varphi}>\frac{\alpha}{\delta} \\
\frac{\beta}{\varphi}>\frac{\gamma}{\varepsilon}
\end{array} \Rightarrow \frac{\alpha}{\delta}>\frac{\gamma}{\varepsilon}(9)\right.\right.\right.
$$

Conditions were obtained for the model's input parameters, the presence of which before the start of the complex of works will allow us to assert that the work will be completed on time and at the appropriate level.

Model 3A

Now let the work not only interfere with each other, because they require simultaneous use of a resource, but at the same time one of the performed works gets a gain in the resource at the expense of the other work. "Internal interference" still remains:

$\left\{\begin{array}{l}\dot{x}=\alpha x-\beta x^{2}-\gamma x y, \\ \dot{y}=\delta y-\varepsilon y^{2}+\varphi x y .\end{array}\right.$

The system has the same attractor as Model 3, but under a lighter condition:

$\alpha \varepsilon-\gamma \delta>0 \Leftrightarrow \frac{\alpha}{\delta}>\frac{\gamma}{\varepsilon}$.

Model 4

Work gets in the way of each other because of the need for a shared resource. However, none of the performed works gets a prize in the resource at the expense of other work. One job doesn't have "internal hindrances", the other has them:

$\left\{\begin{array}{l}\dot{x}=\alpha x-\gamma x y, \\ \dot{y}=\delta y-\varepsilon y^{2}-\varphi x y .\end{array}\right.$

There are no attractors in the system.

Model 4A

In this modification of the model 4 work interferes with each other, and one of the performed jobs gets a gain in the resource at the expense of the other work. This work has "internal hindrances", the other does not:

$\left\{\begin{array}{l}\dot{x}=\alpha x-\gamma x y, \\ \dot{y}=\delta y-\varepsilon y^{2}+\varphi x y .\end{array}\right.$ 
A stable solution with a physical meaning - a nontrivial point-can occur in the system $\left(\frac{\alpha \varepsilon-\gamma \delta}{\gamma \varphi} ; \frac{\alpha}{\gamma}\right)$. The occurrence of such an attractor is provided by the following condition:

$\alpha \varepsilon-\gamma \delta>0 \Rightarrow \frac{\alpha}{\gamma}>\frac{\delta}{\varepsilon}(14)$

Model 4B

Another modification of model 4 is obtained in the same assumptions as model 4A: work interferes with each other, and one of the performed works gains a resource at the expense of another work. But this is no work of "internal interference" in the work of donor they are:

$\left\{\begin{array}{l}\dot{x}=\alpha x+\gamma x y \\ \dot{y}=\delta y-\varepsilon y^{2}-\varphi x y .\end{array}\right.$

The composition of special points in model $4 \mathrm{~B}$ is determined uniquely - all points are unstable. It should be noted that the solutions of model $4 \mathrm{~B}$ are either degenerate or do not make physical sense.

\section{Model 5}

Both jobs have "internal interference", one of the jobs loses its resource due to the fact that another job is performed by it. However, other work does not get any profit in the resource:

$\left\{\begin{array}{l}\dot{x}=\alpha x-\beta x^{2} \\ \dot{y}=\delta y-\varepsilon y^{2}-\varphi x y .\end{array}\right.$

A stable solution with a physical meaning - a nontrivial point-can occur in the system $\left(\frac{\alpha}{\beta} ; \frac{\beta \delta-\alpha \varphi}{\varepsilon \beta}\right)$. The occurrence of such an attractor is provided by the following condition:

$\beta \delta-\alpha \varphi>0 \Rightarrow \frac{\delta}{\varphi}>\frac{\alpha}{\beta}$

\section{Model 5A}

The presented modification of model 5 has a limiting character: in it, the performance of both works is still hindered by "internal interference", but one of them gets a gain in the resource, although the other work does not lose anything in the resources. This is possible, for example, when the share of the resource being pulled is very small in comparison with the amount of the resource allocated to perform the work. Therefore, we can assume that there are no losses in the resource for other work:

$\left\{\begin{array}{l}\dot{x}=\alpha x-\beta x^{2} \\ \dot{y}=\delta y-\varepsilon y^{2}+\varphi x y .\end{array}\right.$
Despite the fact that the model describes a very specific situation, there is always an attractor in the system that has a physical meaning: $\left(\frac{\alpha}{\beta} ; \frac{\beta \delta+\alpha \varphi}{\varepsilon \beta}\right)$.

Model 6

The model describes a situation when one job has internal interference and another has external interference. Moreover, the first job does not win in the resource:

$\left\{\begin{array}{l}\dot{x}=\alpha x-\beta x^{2} \\ \dot{y}=\delta y-\varphi x y\end{array}\right.$

Since we are not interested in the type of trivial singular points, we can say that the set of singular points of model 6 is uniquely defined.

When the $\frac{\alpha}{\beta}=\frac{\delta}{\varphi}$ condition is met, a set of stable States occurs in the system, located on a line defined by the eigenvector of the system for the $\lambda_{1}<0$. It is assumed that the equality of the ratio of the performance of work to the performance of interference for both works ensures that the task is completed with the specified characteristics.

Model 6A

The presented modification of model 6 is as extreme as model 5A: in it, there is a gain in the resource for one job at the expense of another job, and the first job does not lose anything in the resource, but only loses due to internal interference:

$\left\{\begin{array}{l}\dot{x}=\alpha x-\beta x^{2}, \\ \dot{y}=\delta y+\varphi x y .\end{array}\right.$

There are two special points in the model, both trivial: $(0$; $0)$ and $\left(\frac{\alpha}{\beta} ; 0\right)$ - unstable node and saddle, respectively.

\section{CONCLUSION}

Based on the principles of soft modeling, mathematical models were constructed that describe the qualitative effects that occur when a complex of works is performed simultaneously in the conditions of shared resources.

Based on the results of the simulation, the following conclusion can be made.

All the models presented have a trivial special point. In all models, this particular point is of the "unstable node" type, since it is assumed that both works are accepted for execution.

Special points with a single zero coordinate that occur in the models were considered trivial, since we are interested in the fact that both works were performed. 
We considered the existence of a stable singular point with positive coordinates as a condition for the feasibility of the complex of works.

Among the twelve models considered, the feasibility of the work package is possible in seven situations: model 1, model 3, model 3A, model 4A, model 5, model 5A, model 6.

In other cases, the workability is not guaranteed. This means that the management should avoid the situations described by models 2, 2A, 4, 4B, 6A, since the combination of the described factors will not under any circumstances lead to the desired result, and the complex of works will not be performed.

Among the models with a positive outcome, only four models (model 1, model 3A, model 5A, model 6) give an unconditional result: they always have an attractor regardless of the ratio of evolutionary parameters. In the three remaining models (model 3 , model $4 \mathrm{~A}$, and model 5), the positive result is conditional, since certain parameter restrictions must be met to achieve it.

The practical significance of the model is that it allows you to determine the possibility of compatibility of work without quantitative calculations in conditions of limited resources and at the existing level of management. Compatibility is defined as obtaining a result of a certain quality within a given time frame.

Within the framework of the assumptions introduced in the construction of models, it can be argued that all the proposed combinations of factors that affect the intensity of the complex of works are a kind of reflection of the level of management in the organization. The issue of increasing the number of jobs in the model requires further research. According to the current state of Affairs in the field of qualitative theory of differential equations, we can only increase the number of papers to three, since there is a classification of singular points in threedimensional space [8]. The issue of employee motivation modeling was also left aside, since this is a separate large task that is primarily of great practical importance.

\section{ACKNOWLEDGMENT}

The reported study was funded by RFBR and VASS, project number 20-510-92005.

\section{REFERENCES}

[1] N.N. Moiseev, Prostejshie matematicheskie modeli e`konomicheskogo prognozirovaniya, URSS, 2020, Available at: http://bookre.org/reader?file=489196.

[2] G. Emerson, The twelve principles of efficiency, URSS, 2019, Available at: https://pqmonline.com/assets/files/lib/books/emerson.pdf.
[3] L.E. Grinin, A.L. Grinin, The Cybernetic Revolution and the Forthcoming Epoch of SelfRegulating Systems, Moscow branch of Uchitel Publishing House, 2016, Available at: https://search.rsl.ru/ru/record/01008580219.

[4] S.E. Page, The model thinker: what you need to know to make data work for you, Hachette Book Groupe, Inc, 2018, Available at: https://ru.pdfdrive.com/the-model-thinker-what-youneed-to-know-to-make-data-work-for-youe156644755.html.

[5] S.P. Kapicza, S.P. Kurdyumov, G.G. Malineczkij, Sinergetikaiprognozy` budu-shhego, URSS, 2019, Available at: http://spkurdyumov.ru/forecasting/sinergetika-iprognozy-budushhego/.

[6] A.-L. Barabási, The Formula: The Universal Laws of Success, NY: Little, Brown and Company, 2018, Available at:

https://www.academia.edu/40786531/Book_Review_B arabási_L._2018_The_Formula_The_universal_laws_o f_success_2018_.

[7] S. Thurner, P. Klimek, R. Hanel, Introduction to the theory of complex systems, Oxford University Press, 2018, Available at:

https://global.oup.com/academic/product/introductionto-the-theory-of-complex-systems$9780198821939 ? \mathrm{cc}=\mathrm{us} \&$ lang $=\mathrm{en} \&$.

[8] V.I. Arnold,

Oby`knovenny`edifferencial`ny`euravneniya, MCCME 2018, Available at: https://mccme.ru/arnold/books/odu12.pdf.

[9] O.S. Sukharev, Theory of Economic Growth: Institutions, Structures, Technologies, Information, URSS, 2019, Available at: https://search.rsl.ru/ru/record/01009865743.

[10] M.A. Polyanin, Osnovy my`shleniyarukovoditelya: Sistemny`jpodxod k upravleniyudelami v zhiznii v biznese, URSS, 2018, Available at: https://search.rsl.ru/ru/record/01009611279.

[11] A.M. Berdahl, A.B. Kao, A. Flack, P.A.H. Westley, E.A. Codling, I.D. Couzin, A.I. Dell, D. Biro, Collective animal navigation and migratory culture: from theoretical models to empirical evidence, Philosophical Transactions of the Royal Society B - 
Biological Sciences 373(1746) (2018). DOI: 10.1098/rstb.2017.0009

[12] P. Klimek, S. Poledna, S. Thurner, Economic resilience from input-output susceptibility improves predictions of economic growth and recovery, 2019, DOI: 10.1038/s41467-019-09357-w

[13] J. Geanakoplos, K.J. Walsh, Uniqueness and stability of equilibrium in economies with two goods, Journal of Economic Theory 174 (2018) 261-272. DOI: 10.1016/j.jet.2017.12.005

[14] E. Economo, L. Hong, S. E. Page, Social structure, endogenous diversity, and collective accuracy, Journal of Economic Behavior \& Organization 125 (2016) 212-231. DOI:

10.1016/j.jebo.2016.01.003

[15] J.P. O'Dwyer, Whence Lotka-Volterra? Conservation Laws and Integrable Systems in Ecology, Theoretical Ecology, 2018. DOI: 10.1007/s12080-0180377-0

[16] P.J. Lamberson, S.E. Page, First mover or higher quality? Optimal product strategy in markets with positive feedbacks, Journal of Economics \& Management Strategy 27,1 (2018) 40-52. DOI: 10.1111/jems. 12231

[17] J. Garland, A.M. Berdahl, J. Sun, E.M. Bollt, Anatomy of leadership in collective behavior, Chaos 28(7) (2018) 075308. DOI: 10.1063/1.5024395

[18] J. Bendor, S.E. Page, Optimal team composition for tool-based problem solving, Journal of Economics \& Management Strategy 28(5) (2018). DOI:

$10.1111 /$ jems. 12295

[19] O. Peters, M. Gell-Mann, Evaluating gambles using dynamics, Chaos 26(2) (2016). DOI:

10.1063/1.4940236

[20] E.R. Brush, D.C. Krakauer, J.A. Flack, Conflicts of interest improve collective computation of adaptive social structures, Science Advances 4(1) (2018)

1603311. DOI: $10.1126 /$ sciadv.1603311/

[21] P. Schuster, Amathematical model of evolution, Match-Communications in Mathematical and in Computer Chemistry 80(3) (2018) 547-585. DOI: $0.1177 / 053901847501400509$

[22] S. Bowles, The Moral Economy: Why Good Incentives Are No Substitute for Good Citizens, Yale
University Press, 2016, Available at: https://www.moralmarkets.org/book/the-moraleconomy/.

[23] R. Hausmann, F. Neffke, The workforce of pioneer plants: The role of worker mobility in the diffusion of industries, Research Policy 48(3) (2018) 628-648. DOI:10.1016/j.respol.2018.10.017

[24] S. Bowles, The Moral Economy: Why Good Incentives Are No Substitute for Good Citizens, Yale University Press, 2016, Available at: https://www.moralmarkets.org/book/the-moraleconomy/.

[25] W.F. Vining, F. Esponda, M.E. Moses, S. Forrest, How does mobility help distributed systems compute?, Philosophical Transactions of the Royal Society B Biological Sciences, 2019. DOI: 10.1098/rstb.2018.0375. 